\title{
Pre-equilibrium approximation in chemical and photophysical kinetics
}

\author{
Margaret Rae, Mário N. Berberan-Santos* \\ Centro de Química-Física Molecular, Instituto Superior Técnico, 1049-001 Lisboa, Portugal
}

Received 23 January 2002; in final form 25 April 2002

\begin{abstract}
For most mechanisms of chemical reactions and molecular photophysical processes the time evolution of the concentration of the intervening species cannot be obtained analytically. The pre-equilibrium approximation is one of several useful approximation methods that allow the derivation of explicit solutions and simplify numerical solutions. In this work, a general view of the pre-equilibrium approximation is presented, along with the respective analytical solution. It is also shown that the kinetic behavior of systems subject to pre-equilibration can be obtained by the application of perturbation theory. Several photophysical systems are discussed, including excimer formation, thermally activated delayed fluorescence, and external-heavy atom quenching of luminescence. (c) 2002 Elsevier Science B.V. All rights reserved.
\end{abstract}

\section{Introduction}

It is well known that for most mechanisms of chemical reactions and molecular photophysical processes the time evolution of the concentration of the intervening species cannot be obtained explicitly. In order to derive approximate analytical expressions, several classical methods are used, namely the steady-state approximation (SSA), also called quasi-steady-state approximation, and the pre-equilibrium approximation (PEA), also called equilibrium approximation

\footnotetext{
${ }^{*}$ Corresponding author.

E-mail address: berberan@ist.utl.pt (M.N. Berberan-
} Santos).
$[1,2]$. This last approximation is usually invoked when fast reversible reactions precede slower ones in a mechanism [1,2]. Both approximations can also be used to simplify the numerical integration of complex reaction schemes, reducing the dimensionality and decoupling time scales [3].

In recent years, the understanding of these approximations was significantly increased [3-7]. In addition, new numerical methods have been developed for the approximate description of the approach to equilibrium, that is, of asymptotic dynamics [3-5,7]. Of these, the most accurate are typically restricted to systems with a small number of degrees of freedom $[3,7]$.

The kinetics of molecular photophysical processes has a special characteristic: Apart from the intrinsic mechanism of each process, all ex- 
cited species are unstable and decay by radiative and non-radiative unimolecular paths. Usually a small number of species is involved [8-11], but the number of different excited species can also be large. For instance, in the quenching of molecular fluorescence or phosphorescence in concentrated solutions by the external heavy-atom effect, it is possible to have as much as 20 different and interconverting charge-transfer complexes [12].

General quantitative conditions for the applicability of the PEA to these cases, as well as the corresponding general analytical solution under this approximation appear not to be available in the literature, although several particular cases have been considered $[1,2,4,5,8,13]$.

In this work, the goal is to obtain accurate analytical approximations for a relatively general and realistic kinetic scheme under the conditions of applicability of the PEA. This is achieved by means of a vectorial formulation of perturbation theory. The first-order result is also derived by a direct analysis of the rate equations. The obtained results are of interest in ground state reactions and also of direct application in photophysical processes like external-heavy atom quenching in fluid medium [12] and thermally activated delayed fluorescence $[14,15]$.

The outline of this article is as follows. Two important particular cases, the Lindemann scheme and the monomer-excimer scheme, are discussed in Section 2 in connection with the PEA. In Section 3 a more general scheme is considered, and its PEA solution obtained, and applied to external heavy-atom quenching of luminescence. In Section 4 the problem is formulated vectorially, and a perturbational treatment valid for non-symmetric matrices developed. The long-time behavior of quasi pre-equilibrated systems is explicitly obtained up to second order. A general expression for the rate constant is derived, and its applicability conditions discussed. In Section 5 the obtained results are applied to the monomer-excimer scheme, and also discussed in geometrical terms. An interesting result concerning a particular case under very strong perturbations is also obtained. Section 6 contains the final conclusions.

\section{Pre-equilibrium approximation: particular cases}

\subsection{Lindemann-type scheme}

The PEA is often discussed in connection with the mechanism [2,4,13] (Scheme 1) for which an exact explicit solution exists. Since this mechanism is only composed by unimolecular steps, the solution can be obtained by the standard eigenvalue method $[2,16]$.

The time evolution of any of the participating species is given by a sum of three exponentials, whose arguments contain the eigenvalues of the kinetic matrix. One of the eigenvalues, corresponding to the long-time limit (true equilibrium) is zero, while the other two are negative, and given by algebraic expressions involving the rate constants of the elementary steps $[8,13]$.

This mechanism applies to several real cases, namely acid-base equilibrium preceding a first-order or pseudo first-order step [1], and is also the simplest model for enzyme-catalyzed reactions (Henri-Michaelis-Menten mechanism [1,2,4,5]), unimolecular reactions in the gas phase (Lindemann mechanism [1,2]), and diffusion-influenced reactions [1,2]. Conditions of applicability of the PEA approximation to this mechanism have been discussed in detail $[4,13]$. It was namely found that the PEA applies if $k_{12}+k_{21} \gg \Gamma$ and this only after an induction period (equilibration time) of $1 /\left(k_{12}+k_{21}\right)$.

Application of the PEA yields a common rate constant describing the long-time evolution of all three species $A_{1}, A_{2}$, and $C$,

$$
\begin{aligned}
& {\left[A_{1}\right]=\frac{k_{21}}{k_{12}+k_{21}}\left[A_{1}\right]_{0} \mathrm{e}^{-k t},} \\
& {\left[A_{2}\right]=\frac{k_{12}}{k_{12}+k_{21}}\left[A_{1}\right]_{0} \mathrm{e}^{-k t},} \\
& {[C]=\left[A_{1}\right]_{0}\left(1-\mathrm{e}^{-k t}\right),}
\end{aligned}
$$

$$
A_{1} \underset{k_{21}}{\stackrel{k_{12}}{\rightleftarrows}} A_{2} \stackrel{\Gamma}{\longrightarrow} C
$$

Scheme 1. 
where

$k=\frac{k_{12} \Gamma}{k_{12}+k_{21}}$,

it being assumed that $\left[A_{2}\right]_{0}=[C]_{0}=0$. The overall rate constant $k$ can be rewritten as,

$k=x_{2} \Gamma$,

i.e., it is the product of the molar fraction of the active reagent molecules $\left(A_{2}\right), x_{2}$, given by $k_{12} /\left(k_{12}+k_{21}\right)$, by the intrinsic rate constant $(\Gamma)$.

Under the conditions of applicability of the PEA, the two eigenvalues of the system are $-\left(k_{12}+k_{21}\right)$ and $-k$. One has $\left(k_{12}+k_{21}\right)>>k$, hence, as discussed, after a short period of time, dominated by $k_{12}+k_{21}$, and corresponding to preequilibration of $A_{1}$ and $A_{2}$, the system slowly evolves according to the smaller (in modulus) eigenvalue, $-k$. It is under this regime that the PEA is valid. We defer the discussion of the mechanism in geometrical terms until Section 5 .

\subsection{Monomer-excimer type scheme}

A slightly more complex mechanism is given in Scheme 2.

This mechanism applies, for instance, to monomer-excimer kinetics, where $A_{1}$ is an excited monomer and $A_{2}$ is an excimer. The exact, explicit solution is again known $[8,10,13]$. In the monomer-excimer case, the situation where the PEA is valid corresponds to the so-called high-temperature behavior [8], where a fast equilibrium in the excited state exists, and for which a single exponential decay (after a short induction period) with a common decay time for both monomer and excimer is predicted, and experimentally observed [8]. Application of the PEA approximation to Scheme 2 yields

$\left[A_{1}\right]=\frac{k_{21}}{k_{12}+k_{21}}\left[A_{1}\right]_{0} \mathrm{e}^{-k t}$,

$$
C_{1} \stackrel{\Gamma_{1}}{\longleftarrow} A_{1} \stackrel{k_{12}}{\underset{k_{21}}{\rightleftarrows}} A_{2} \stackrel{\Gamma_{2}}{\longrightarrow} C_{2}
$$

Scheme 2.

$$
\begin{aligned}
& {\left[A_{2}\right]=\frac{k_{12}}{k_{12}+k_{21}}\left[A_{1}\right]_{0} \mathrm{e}^{-k t},} \\
& {\left[C_{1}\right]=\frac{k_{21} \Gamma_{1}}{k_{12} \Gamma_{1}+k_{21} \Gamma_{2}}\left[A_{1}\right]_{0}\left(1-\mathrm{e}^{-k t}\right),} \\
& {\left[C_{2}\right]=\frac{k_{12} \Gamma_{2}}{k_{12} \Gamma_{2}+k_{21} \Gamma_{1}}\left[A_{1}\right]_{0}\left(1-\mathrm{e}^{-k t}\right),}
\end{aligned}
$$

where

$k=\frac{k_{21} \Gamma_{1}+k_{12} \Gamma_{2}}{k_{12}+k_{21}}$,

it being assumed that $\left[A_{2}\right]_{0}=\left[C_{1}\right]_{0}=\left[C_{2}\right]_{0}=0$. The rate constant $k$, given by Eq. (2.10), can be rewritten as,

$k=x_{1} \Gamma_{1}+x_{2} \Gamma_{2}$,

i.e., it is the weighted sum of the rate constants $\Gamma_{1}$ and $\Gamma_{2}$ of the two consumption paths, the weighting factors being the molar fractions of the reacting species ( $A_{1}$ and $A_{2}$, respectively) in each of the paths. The discussion of the mechanism in geometrical terms is again postponed until Section 5.

\subsection{Thermally activated delayed fluorescence}

Consider now thermally activated delayed fluorescence kinetics, depicted in Scheme 3.

Where the following three inequalities are usually obeyed: $k_{\mathrm{ST}} \gg k_{\mathrm{SG}} ; k_{\mathrm{TS}} \gg k_{\mathrm{TG}}$; and $k_{\mathrm{ST}} \gg k_{\mathrm{TS}}$. In this phenomenon, exhibited by some dyes [14] and fullerenes [15], interconversion of the singlet and triplet emissive states occurs many times, before photon emission or non-radiative decay can take place. There is therefore a fast pre-equilibrium between $S_{1}$ and $T_{1}$. The kinetic scheme is seen to be identical to the monomer-excimer one discussed above. In this way, a common rate constant

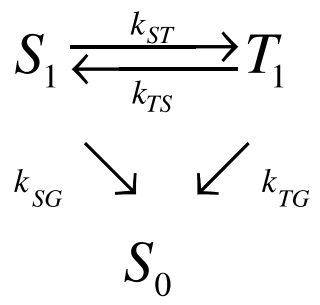

Scheme 3. 
for the decay of both $S_{1}$ and $T_{1}$ is given by Eq. (2.12),

$k=\frac{k_{\mathrm{ST}}}{k_{\mathrm{ST}}+k_{\mathrm{TS}}} k_{\mathrm{TG}}+\frac{K_{\mathrm{TS}}}{k_{\mathrm{ST}}+k_{\mathrm{TS}}} k_{\mathrm{SG}}$.

Given the inequalities mentioned, Eq. (2.12) simplifies to

$k=k_{\mathrm{TG}}+\left(1-\Phi_{\mathrm{T}}\right) k_{\mathrm{TS}}$,

where $\Phi_{\mathrm{T}}$ is the quantum yield of triplet formation, $\Phi_{\mathrm{T}}=k_{\mathrm{ST}} /\left(k_{\mathrm{ST}}+k_{\mathrm{SG}}\right)$. In this way, the slow decay constant, common to fluorescence and phosphorescence, is close to the triplet intrinsic decay $\left(k_{\mathrm{TG}}\right)$.

\section{General case}

\subsection{General result}

One might now consider schemes of increasing complexity, like Scheme 4 or Scheme 5 and obtain the respective PEA results.

Instead, let us consider immediately the general case of a fast pre-equilibrium (by means of internal steps) involving a number of species $A_{i}(i=1,2, .$. , $n$ ), each of which may in turn react to yield other species not participating in the fast pre-equilibrium, by means of outgoing steps, as shown in Scheme 6.

It is assumed that the internal steps are unimolecular or pseudo-unimolecular of the type

$A_{i}+B_{j} \leftrightarrows A_{k}+B_{l}$

where the $B_{i}$ are (eventually) present but only in these internal steps, and have essentially constant

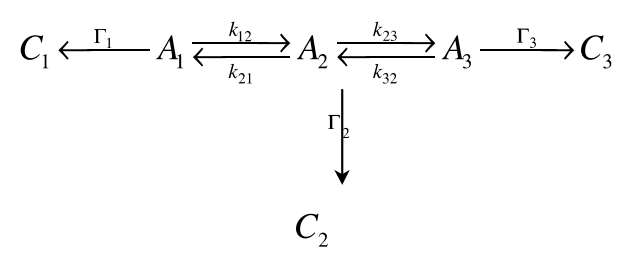

Scheme 4.

$$
A_{1}+B_{1} \underset{k_{21}}{\stackrel{k_{12}}{\rightleftarrows}} B_{2}+A_{2} \stackrel{\Gamma}{\longrightarrow} C
$$

Scheme 5.

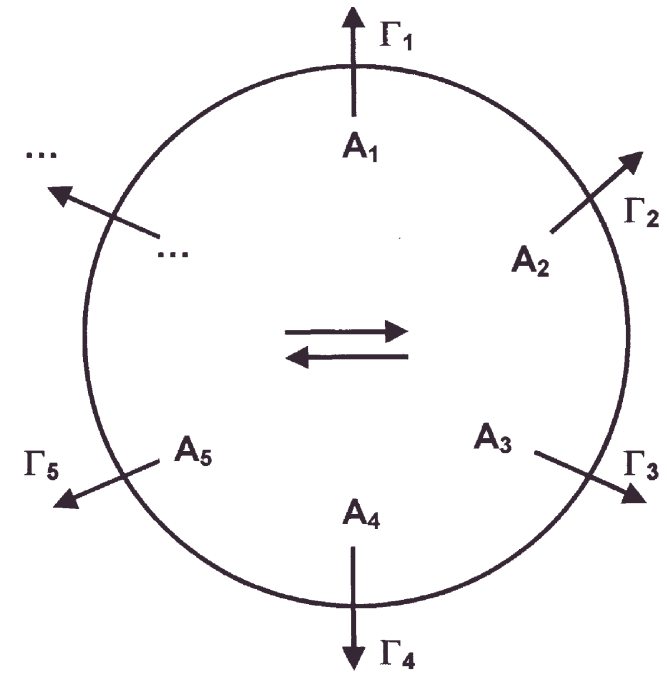

Scheme 6.

concentration, while the $A_{i}$ participate also in outgoing steps of the type

$A_{i} \rightarrow C_{i}+\cdots$

This scheme corresponds for instance to the quenching of molecular fluorescence or phosphorescence in concentrated fluid solutions by the external heavy-atom effect, where a large number of different and rapidly interconverting luminescent complexes may exist [12]. The still more general case where outgoing bimolecular steps are allowed is discussed in Appendix A.

Based on the special results discussed in Section 2 , it seems reasonable to suppose that the general form of the long-time rate constant $k$ obtained by the PEA will be

$k=\sum_{i=1}^{n} x_{i} \Gamma_{i}$

where the $x_{i}$ are the equilibrium molar fractions of the $A_{i}$

$x_{i}=\frac{\left[A_{i}\right]}{\sum_{i=1}^{n}\left[A_{i}\right]}$,

and the $\Gamma_{i}$ are the respective rate constants for the outgoing, irreversible steps, as shown in Scheme 6. The molar fractions $x_{i}$ can be related to the rate constants of the internal steps, as previously shown in Section 2. 
A simple proof that Eq. (3.1) is indeed correct is now given.

For any of the $A_{i}(i=1,2, \ldots, n)$ of Scheme 6 , the following rate equation holds,

$\frac{\mathrm{d}\left[A_{i}\right]}{\mathrm{d} t}=r_{i}-\Gamma_{i}\left[A_{i}\right] \quad(i=1,2, \ldots, n)$,

where $r_{i}$ is the global rate for $A_{i}$ resulting from all internal steps (unimolecular or bimolecular). Summing all rate Eq. (3.3), one gets

$\frac{\mathrm{d} \sum_{i=1}^{n}\left[A_{i}\right]}{\mathrm{d} t}=\sum_{i=1}^{n} r_{i}-\sum_{i=1}^{n} \Gamma_{i}\left[A_{i}\right]$.

Now, $\sum_{i=1}^{n} r_{i}=0$ for all times, since the total number of moles of the $A_{i}$ is conserved for internal reactions, and Eq. (3.4) becomes

$\frac{\mathrm{d} \sum_{i=1}^{n}\left[A_{i}\right]}{\mathrm{d} t}=-\sum_{i=1}^{n} \Gamma_{i}\left[A_{i}\right]$.

After the time lapse needed for equilibration, Eq. (3.2) must hold, and Eq. (3.5) becomes

$\frac{\mathrm{d} \sum_{i=1}^{n}\left[A_{i}\right]}{\mathrm{d} t}=-\left(\sum_{i=1}^{n} x_{i} \Gamma_{i}\right)\left(\sum_{i=1}^{n}\left[A_{i}\right]\right)$.

Since the equilibrium molar fractions $x_{i}$ are timeindependent, a single exponential decay of $\sum_{i=1}^{n}\left[A_{i}\right]$ follows from Eq. (3.6)

$\sum_{i=1}^{n}\left[A_{i}\right]=\left(\sum_{i=1}^{n}\left[A_{i}\right]_{\mathrm{eq}}\right) \exp (-k t)$,

where the rate constant $k$ is given by Eq. (3.1), and $\left[A_{i}\right]_{\mathrm{eq}}$ stands for the concentration of $A_{i}$ upon full equilibration, in the absence of outgoing steps. Since, from Eq. (3.2), $\left[A_{i}\right]$ is proportional to $\sum_{i=1}^{n}\left[A_{i}\right]$, one finally has

$\left[A_{i}\right]=x_{i}\left(\sum_{i=1}^{n}\left[A_{i}\right]_{\mathrm{eq}}\right) \exp (-k t) \quad(i=1,2, \ldots, n)$,

again with $k$ given by Eq. (3.1).

Note that it cannot be assumed that after the equilibration time one will have exactly $r_{i}=0(i=1,2, \ldots, n)$ in Eq. (3.3), otherwise the equilibrium mole fractions would not be time-independent, since the disappearance rates $\Gamma_{i}$ are in general different for each species. This observation serves to stress that the fast equilibration not only occurs at the early stages of the reaction, just before the PEA becomes valid, but keeps going on at latter times, in order to maintain a state of (dynamic) equilibrium.

The validity of Eq. (3.8) rests on the assumption of a fast pre-equilibration process, with respect to the characteristic times of the outgoing processes. These are given by the inverse of the rate constants $\Gamma_{i}$. The internal pre-equilibrium must therefore be attained in a time considerably shorter than the smallest $1 / \Gamma_{i}$.

\subsection{Application to external heavy-atom quenching in fluid solution}

Heavy-atom quenching of fluorescence is explained by an increase in the probability of the $S_{1} \rightarrow T_{n}$ radiationless transition of the fluorophore. Heavy-atom quenching of fluorescence may occur in two different ways. Firstly, the heavyatom can be part of the chromophore under study; this is referred to as the internal heavy-atom effect. If however the heavy-atom quencher is not part of the chromophore, then the effect is said to be external. This process requires close contact between perturber and fluorophore, usually in the form of a statistical charge-transfer complex (exciplex with essentially zero binding energy). In fluid media, this complex is short-lived, as it is both brought about and broken apart by the incessant diffusive motion of quencher and fluorophore.

For the external heavy-atom quenching of the fluorescence of an excited molecule $M^{*}$ by a relatively dilute solution of quencher $Q$ in a fluid medium, the mechanism shown in Scheme 7 is considered, where $k_{d}$ and $k_{-d}$ are the rate constants for diffusion controlled association and dissociation, respectively, and $\Gamma_{0}$ and $\Gamma_{1}$ are the decay rates of the free fluorophore and of the fluorophore in the charge-transfer complex, i.e., when subject to the external heavy-atom effect. It is assumed that no stable complex between the ground or excited state fluorophore and $Q$ exists. $\left(M^{*} Q\right)$ is therefore a statistical complex. When, as is usually the case, $[Q] \gg$ $[M]$, Scheme 7 becomes identical to the monomerexcimer one, Scheme 2. In most cases, the dissoci- 


$$
\stackrel{\Gamma_{0}}{\longleftarrow} M^{*}+Q \underset{k_{-d}}{\stackrel{k_{d}}{\rightleftarrows}}\left(M^{*} Q\right) \stackrel{\Gamma_{1}}{\longrightarrow}
$$

Scheme 7.

ation rate constant of the complex is much higher than the decay rate of the perturbed fluorophore, $k_{-d} \gg \Gamma_{1}$, i.e., the perturbed fluorophore lifetime greatly exceeds that of the momentary complex. For a solvent with a viscosity similar to that of a light alkane or water, and at room temperature, $k_{-d} \gg \Gamma_{1}$ imposes on the perturbed fluorophore lifetime a lower limit of a few hundred picoseconds. In such a case, the PEA can be invoked, and the decay of fluorescence is single-exponential, with a rate constant given by Eq. (2.10) or (2.11).

For concentrated quencher solutions, Scheme 7 is no longer satisfactory, because charge-transfer complexes of the type $\left(M^{*} Q_{n}\right)$, with $n>1$ (up to $n=20$ or so, depending on the concentration and species involved), exist in significant amounts [12]. If these complexes are in fast equilibrium, then the general result Eq. (3.1) applies, and the fluorescence decay rate constant is a simple average of those of the $\left(M^{*} Q_{n}\right)$. Using [12]

$\Gamma_{n}=\Gamma_{0}+n \Delta k_{\mathrm{ISC}}$,

where $\Delta k_{\mathrm{ISC}}$ is the increment in the intersystem crossing rate constant for the 1:1 complex, it follows that the fluorescence decay rate constant is

$\Gamma=\Gamma_{0}+\bar{n} \Delta k_{\mathrm{ISC}}$

$\bar{n}$ being the average number of quenchers around $M^{*}, \bar{n}=V_{\mathrm{m}}[Q]$, where $V_{\mathrm{m}}$ is a molar volume close to that of the complex $\left(M^{*} Q\right)$ [12].

\section{Higher-order approximations and systems not fully pre-equilibrated}

A more general approach to the problem consists in writing the general rate equation in matrix form $[3,16,17]$,

$\frac{\mathrm{d}}{\mathrm{d} t}|A\rangle=\mathbf{A}|A\rangle$,

where $|A\rangle$ is the concentration column vector (concentration ket) of the $A_{i}$, and $\mathbf{A}$ is the timeindependent rate matrix,
$\mathbf{A}=\mathbf{K}+\Gamma$,

where the real but usually non-symmetric matrix $\mathbf{K}$ corresponds to the internal steps, and the matrix $\Gamma$ is diagonal and corresponds to the outgoing steps (see Scheme 6).

In the absence of outgoing steps, one has

$\frac{\mathrm{d}}{\mathrm{d} t}|A\rangle=\mathbf{K}|A\rangle$.

The solution to this equation corresponds to the usual relaxation equation $[1,2]$

$|A\rangle=\sum_{i} a_{i} \mathrm{e}^{\lambda_{i} t}\left|\lambda_{i}\right\rangle$,

where the $a_{i}$ are scalar coefficients determined by the initial concentrations, the $\left|\lambda_{i}\right\rangle$ are eigenvectors of $\mathbf{K}$, and the $\lambda_{i}$ are the corresponding eigenvalues,

$\mathbf{K}\left|\lambda_{i}\right\rangle=\lambda_{i}\left|\lambda_{i}\right\rangle$.

The relaxation times of the system are $\tau_{i}=-1 / \lambda_{i}$. Non-degenerate eigenvalues are assumed throughout. As physically required, all eigenvalues are nonpositive. Usually, only one eigenvalue $\left(\lambda_{0}\right)$ is zero, whose eigenvector defines the straight line, where equilibrium solutions lie. When this eigenvector is appropriately scaled according to the initial conditions, it defines the point corresponding to the particular equilibrium solution. This point is an attractor of the system. The eigenvectors define the characteristic directions of relaxation towards the final equilibrium state. Any reaction path is defined by a time-dependent vector that is the weighted sum of these vectors, cf. Eq. (4.4).

The eigenvalue Eq. (4.5) can also be written,

$\left\langle\lambda_{i}\right| \mathbf{K}=\lambda_{i}\left\langle\lambda_{i}\right|$,

where $\left\langle\lambda_{i}\right|$ is the row concentration vector (concentration bra). Since $\mathbf{K}$ is in general non-symmetric, the bra eigenvectors are not simply the transpose of the corresponding kets. The eigenvectors may however be still chosen so as to obey the orthonormality condition

$\left\langle\lambda_{i} \mid \lambda_{j}\right\rangle=\delta_{i j}$.

The matrix $\mathbf{K}$ can then be written

$\mathbf{K}=\sum_{i} \lambda_{i}\left|\lambda_{i}\right\rangle\left\langle\lambda_{i}\right|$ 
We now consider the effect of the introduction of the outgoing steps, represented by the diagonal matrix $\Gamma$. The solution of Eq. (4.1) is formally identical to that of Eq. (4.3), i.e., of the form

$\left|A^{\prime}\right\rangle=\sum_{i} a_{i}^{\prime} \mathrm{e}^{\lambda_{i}^{\prime} t}\left|\lambda_{i}^{\prime}\right\rangle$.

Under the PEA, the outgoing steps are regarded as a small perturbation to the kinetics of the system. In order to relate $\lambda_{i}^{\prime}$ with $\lambda_{i}$ a perturbational approach is therefore appropriate. The eigenvalue equation is

$(\mathbf{K}+\Gamma)\left|\lambda_{i}^{\prime}\right\rangle=\lambda_{i}^{\prime}\left|\lambda_{i}^{\prime}\right\rangle$.

The perturbed solution can be written in terms of the basis (complete set of eigenvectors) of the unperturbed system

$\left|\lambda_{i}^{\prime}\right\rangle=\sum_{j} c_{i j}\left|\lambda_{j}\right\rangle$

and using Eqs. (4.5), (4.10) becomes

$\sum_{j} c_{i j} \lambda_{j}\left|\lambda_{j}\right\rangle+\sum_{j} c_{i j} \Gamma\left|\lambda_{j}\right\rangle=\lambda_{i}^{\prime} \sum_{j} c_{i j}\left|\lambda_{j}\right\rangle$.

Multiplication of Eq. (4.12) by $\left\langle\lambda_{i}\right|$ yields

$c_{i i} \lambda_{i}+\sum_{j} c_{i j}\left\langle\lambda_{i}|\Gamma| \lambda_{j}\right\rangle=\lambda_{i}^{\prime} c_{i i}$

which, upon rearrangement, gives

$\lambda_{i}^{\prime}=\lambda_{i}+\frac{1}{c_{i i}} \sum_{j} c_{i j}\left\langle\lambda_{i}|\Gamma| \lambda_{j}\right\rangle$.

One has, in all orders of approximation, $c_{i i} \cong 1$, hence Eq. (4.14) becomes

$\lambda_{i}^{\prime}=\lambda_{i}+\sum_{j} c_{i j}\left\langle\lambda_{i}|\Gamma| \lambda_{j}\right\rangle$.

In the first-order approximation, the zero-order coefficients are used,

$c_{i j}=\delta_{i j}$,

hence, the eigenvalues with corrections up to first order are

$\lambda_{i}^{\prime}=\lambda_{i}+\left\langle\lambda_{i}|\Gamma| \lambda_{i}\right\rangle$.

In the PEA, one would expect to have $\left\langle\lambda_{i}|\Gamma| \lambda_{i}\right\rangle \ll \lambda_{i}$, except for the zero eigenvalue. Hence, $\lambda_{i}^{\prime} \cong \lambda_{i}$, except for $\lambda_{i}=0$. The internal relaxation kinetics is practically unaffected, but instead of an equilibrium solution, the outgoing steps imply a slow decay of all concentrations to zero. The zero eigenvalue is thus replaced by $\langle 0|\Gamma| 0\rangle$.

In order to obtain the explicit form of this eigenvalue, it is noted that $|0\rangle$ is the column eigenvector corresponding to the unperturbed equilibrium solution. It may be chosen so that its elements are the equilibrium concentrations of the $A_{i}$. With this choice, the row eigenvector $\langle 0|$ is (see Appendix B)

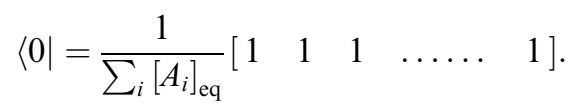

In this way, one finally has

$$
\langle 0|\Gamma| 0\rangle=-\frac{\sum_{i}\left[A_{i}\right]_{\mathrm{eq}} \Gamma_{i}}{\sum_{i}\left[A_{i}\right]_{\mathrm{eq}}}=-\sum_{i} x_{i} \Gamma_{i},
$$

in agreement with Eq. (3.1).

Multiplication of Eq. (4.12) by $\left\langle\lambda_{k}\right|$ yields, upon rearrangement

$c_{i k}^{\prime}=\frac{\sum_{j} c_{i j}\left\langle\lambda_{k}|\Gamma| \lambda_{j}\right\rangle}{\lambda_{i}^{\prime}-\lambda_{k}} \quad(i \neq k)$,

and using again Eq. (4.16) and $\lambda_{i}^{\prime}=\lambda_{i}$ (zero-order values) on the r.h.s., the first-order coefficients become

$c_{i k}^{\prime}=\frac{\left\langle\lambda_{k}|\Gamma| \lambda_{i}\right\rangle}{\lambda_{i}-\lambda_{k}} \quad(i \neq k)$.

By the insertion of Eq. (4.21) into Eq. (4.15), the eigenvalues with correction up to second order are obtained,

$\lambda_{i}^{\prime}=\lambda_{i}+\left\langle\lambda_{i}|\Gamma| \lambda_{i}\right\rangle+\sum_{j \neq i} \frac{\left\langle\lambda_{i}|\Gamma| \lambda_{j}\right\rangle\left\langle\lambda_{j}|\Gamma| \lambda_{i}\right\rangle}{\lambda_{i}-\lambda_{j}}$.

Note that $\left\langle\lambda_{i}|\Gamma| \lambda_{j}\right\rangle$ and $\left\langle\lambda_{j}|\Gamma| \lambda_{i}\right\rangle(i \neq j)$ are in general different.

The eigenvectors are, up to first order,

$\left|\lambda_{i}^{\prime}\right\rangle=\left|\lambda_{i}\right\rangle+\sum_{j \neq i} \frac{\left\langle\lambda_{j}|\Gamma| \lambda_{i}\right\rangle}{\lambda_{i}-\lambda_{j}}\left|\lambda_{j}\right\rangle$.

For the smallest (in absolute value) eigenvalue, Eq. (4.22) reduces to

$\lambda_{0}^{\prime}=\langle 0|\Gamma| 0\rangle-\sum_{j>0} \frac{\left\langle 0|\Gamma| \lambda_{j}\right\rangle\left\langle\lambda_{j}|\Gamma| 0\right\rangle}{\lambda_{j}}$. 
The applicability of a perturbational treatment lies on the smallness of the coefficients $c_{i j}$. From Eq. (4.21), this implies that

$$
\left|\left\langle\lambda_{j}|\Gamma| \lambda_{i}\right\rangle\right| \ll\left|\lambda_{i}-\lambda_{j}\right| \quad(i \neq j),
$$

and this is a supplementary condition for the applicability of the PEA.

It is interesting to consider the application of these results to the simple monomer-excimer scheme.

\section{Monomer-excimer type scheme}

For the monomer-excimer type Scheme 2 discussed in Section 2.2, the rate matrix is

$\mathbf{K}=\left(\begin{array}{cc}-k_{12} & k_{21} \\ k_{12} & -k_{21}\end{array}\right)$,

and the eigenvalues are

$\lambda_{0}=0$,

$\lambda_{1}=-\left(k_{12}+k_{21}\right)$,

while the eigenkets can be chosen to be

$$
\begin{aligned}
& |0\rangle=\left[\begin{array}{c}
1 \\
K
\end{array}\right], \\
& |1\rangle=\left[\begin{array}{c}
1 \\
-1
\end{array}\right],
\end{aligned}
$$

where $K=k_{12} / k_{21}$. With the above choice of eigenkets, the eigenbras are

$$
\begin{aligned}
& \langle 0|=\frac{1}{1+K}\left[\begin{array}{ll}
1 & 1
\end{array}\right], \\
& \langle 1|=\frac{1}{1+K}\left[\begin{array}{ll}
K & -1
\end{array}\right] .
\end{aligned}
$$

The perturbing matrix is

$$
\Gamma=\left(\begin{array}{cc}
-\Gamma_{1} & 0 \\
0 & -\Gamma_{2}
\end{array}\right) .
$$

The matrix elements are:

$$
\begin{aligned}
& \langle 0|\Gamma| 0\rangle=-\frac{\Gamma_{1}+K \Gamma_{2}}{1+K}=-\left(x_{1} \Gamma_{1}+x_{2} \Gamma_{2}\right), \\
& \langle 1|\Gamma| 1\rangle=-\frac{\Gamma_{2}+K \Gamma_{1}}{1+K}=-\left(x_{2} \Gamma_{1}+x_{1} \Gamma_{2}\right),
\end{aligned}
$$

$$
\begin{aligned}
& \langle 0|\Gamma| 1\rangle=\frac{\Gamma_{2}-\Gamma_{1}}{1+K}=x_{1}\left(\Gamma_{2}-\Gamma_{1}\right), \\
& \langle 1|\Gamma| 0\rangle=\frac{K\left(\Gamma_{2}-\Gamma_{1}\right)}{1+K}=x_{2}\left(\Gamma_{2}-\Gamma_{1}\right),
\end{aligned}
$$

hence, up to second order,

$\lambda_{0}^{\prime}=-\frac{\Gamma_{1}+K \Gamma_{2}}{1+K}+\frac{K\left(\Gamma_{2}-\Gamma_{1}\right)^{2}}{(1+K)^{2} \lambda_{1}}$

or

$\lambda_{0}^{\prime}=-\left(x_{1} \Gamma_{1}+x_{2} \Gamma_{2}\right)+x_{1} x_{2} \frac{\left(\Gamma_{2}-\Gamma_{1}\right)^{2}}{\lambda_{1}}$,

and, up to first order,

$\left|0^{\prime}\right\rangle=|0\rangle+\frac{x_{2}\left(\Gamma_{2}-\Gamma_{1}\right)}{\lambda_{1}}|1\rangle$

$\left|1^{\prime}\right\rangle=|1\rangle+\frac{x_{1}\left(\Gamma_{2}-\Gamma_{1}\right)}{-\lambda_{1}}|0\rangle$.

From Eq. (5.14), it is clear that under the PEA, the second-order correction term is usually negligible.

An interesting aspect that is observed in Eqs. (5.14)-(5.16) is the dependence of all higher-order corrections on the difference $\Gamma_{2}-\Gamma_{1}$. If these two rate constants are exactly equal, which is physically unlikely, but mathematically possible, the (long-time) equilibrium position and the initial relaxation path are not affected by the perturbation, even when these rate constants are comparable to or larger than the internal ones.

We conclude this section with a geometrical interpretation of the PEA, as applied to the monomer-excimer scheme, having in mind a previous detailed analysis of the related Lindemann scheme [4]. As discussed, in order for the PEA to be valid, one must have $k \ll\left|\lambda_{1}\right|$. In this case, the reaction path in the phase plane will be essentially a broken line composed by two joined linear segments: one along the direction defined by $|1\rangle$, starting in the initial composition and ending at the equilibrium line (defined by $|0\rangle$ ), and a second one along that line, connecting the intersection point with the origin. The first straight path (internal relaxation) will occur on a comparatively short time, dictated by $\lambda_{1}$, while the second straight path (decay to zero under internal equi- 
librium conditions) will occur on a much longer time, dictated by $k$, i.e., by the outgoing rates and by the equilibrium composition. A magnification of the small region where the two straight segments meet, will show a curved line smoothly connecting them.

The equilibrium line (with slope $K$ ), defined by $|0\rangle$, is confined between two other straight lines, sharing a common point (the origin). The upper one (with slope $K+\Gamma_{1} / k_{21}$ ) corresponds to the SSA applied to $A_{1}$, while the lower one (with slope $K /\left(1+\left(\Gamma_{2} / k_{21}\right)\right)$ ) corresponds to the SSA applied to $A_{2}$. When $k_{12} \gg \Gamma_{1}$, the upper SSA coincides with the PEA line. When $k_{21} \gg \Gamma_{2}$, the lower SSA line coincides with the PEA. Since $k_{12}+k_{21} \gg \Gamma_{1}+\Gamma_{2}$ for the PEA to be valid, there will always be at least one SSA simultaneously obeyed, and only two distinct lines will exist. This appears to be in contrast with Scheme 1, where the two approximations (EA and SSA) not always apply simultaneously. Nevertheless, this obviously occurs because in that scheme (which is a particular case of Scheme 2 with $\Gamma_{1}=0$ ), the upper SSA line, which coincides with the PEA line, is not recognized as such. The SSA is usually not applied to $A_{1}$, since this is not a transient species, and the only SSA line considered corresponds to the lower line of Scheme 2.

It is also important to note that while for Scheme 1 the PEA line is obtained by exactly the same mathematical procedure as that of the (upper) SSA [4], in a more general scheme, like Scheme 2, this is no longer the situation, and the PEA cannot be generally equated with the SSA. There is therefore in general a specific line for the PEA. This conclusion should be stressed, since it is not clear from previous analysis, mainly devoted to the SSA.

\section{Conclusions}

In this work, the goal has been to obtain accurate analytical approximations for a relatively general and realistic kinetic scheme under the conditions of applicability of the PEA. This was achieved by means of a vectorial formulation of perturbation theory. The first-order result was confirmed by a direct analysis of the rate equations. Application of the results to a simple scheme (monomer-excimer) produced some interesting results, namely that: (i) under specific conditions (decay rates identical for all species) the perturbation, however strong, does not affect the final path to equilibrium, and (ii) the PEA line in phase space is in general different from the SSA lines.

The main results obtained, namely Eqs. (3.1), (4.24) and (A.4), are of interest in ground state reactions but are also of direct application in photophysical processes like external-heavy atom quenching in fluid medium and thermally activated delayed fluorescence.

\section{Acknowledgements}

This work was supported by the POCTI program (contract 34400/QUI/2000, FCT, Portugal) and by the TMR program of European Union (research network contract ERB FMRX-CT 980192 DG 12-DLCL).

\section{Appendix A. Inclusion of outgoing bimolecular steps}

A more general situation can be envisaged if outgoing bimolecular steps of the following type

$A_{i}+A_{j} \rightarrow C_{k}+\cdots$

are also allowed. Eq. (3.3) becomes

$$
\begin{gathered}
\frac{\mathrm{d}\left[A_{i}\right]}{\mathrm{d} t}=r_{i}-\left(\sum_{j=1}^{n} k_{i j}\left[A_{j}\right]\right)\left[A_{i}\right]-\Gamma_{i}\left[A_{i}\right] \\
(i=1,2, . ., n)
\end{gathered}
$$

and Eq. (3.4) becomes

$$
\begin{aligned}
\frac{\mathrm{d} \sum_{i=1}^{n}\left[A_{i}\right]}{\mathrm{d} t}= & \sum_{i=1}^{n} r_{i}-\sum_{i=1}^{n} \sum_{j=1}^{n} k_{i j}\left[A_{i}\right]\left[A_{j}\right] \\
& -\sum_{i=1}^{n} \Gamma_{i}\left[A_{i}\right] .
\end{aligned}
$$


Instead of Eq. (3.6), one now has,

$$
\begin{aligned}
\frac{\mathrm{d} \sum_{i=1}^{n}\left[A_{i}\right]}{\mathrm{d} t}= & -\left(\sum_{i=1}^{n} \sum_{j=1}^{n} x_{i} x_{j} k_{i j}\right)\left(\sum_{i=1}^{n}\left[A_{i}\right]\right)^{2} \\
& -\left(\sum_{n=1}^{n} x_{i} \Gamma_{i}\right)\left(\sum_{i=1}^{n}\left[A_{i}\right]\right)
\end{aligned}
$$

whose solution is

$$
[A]=\frac{[A]_{\mathrm{eq}}}{\left(1+\frac{k_{2}[A]_{\mathrm{eq}}}{k_{1}}\right) \exp \left(k_{1} t\right)-\frac{k_{2}[A]_{\mathrm{eq}}}{k_{1}}},
$$

where

$$
[A]=\sum_{i=1}^{n}\left[A_{i}\right],[A]_{\mathrm{eq}}=\sum_{i=1}^{n}\left[A_{i}\right]_{\mathrm{eq}},
$$

and

$$
\begin{aligned}
& k_{1}=\sum_{i=1}^{n} x_{i} \Gamma_{i}, \\
& k_{2}=\sum_{i=1}^{n} \sum_{j=1}^{n} x_{i} x_{j} k_{i j} .
\end{aligned}
$$

If $k_{2}=0$, Eq. (A.4) reduces to Eq. (3.8), which has the form of the elementary first order reaction $A \rightarrow P$. If, on the other hand, $k_{1}=0$, then Eq. (A.4) becomes

$$
[A]=\frac{[A]_{\mathrm{eq}}}{1+k_{2}[A]_{\mathrm{eq}} t},
$$

which has the form of the elementary second order reaction $2 A \rightarrow P$. The more general Eq. (A.4) corresponds to mixed first and second order kinetics.

\section{Appendix B. Derivation of Eq. (4.18)}

The rate matrix $\mathbf{K}$ of the unperturbed scheme is

$\mathbf{K}=\left(\begin{array}{cccc}-k_{1} & k_{21} & \cdots & k_{n 1} \\ k_{12} & -k_{2} & \cdots & k_{n 2} \\ \cdots & \cdots & \cdots & \cdots \\ k_{1 n} & k_{2 n} & \cdots & -k_{n n}\end{array}\right)$ where the diagonal elements are

$k_{i}=\sum_{j \neq i} k_{i j}$

and the $k_{i j}$ are the rate constants of the elementary steps. For the zero eigenvalue, the left eigenvalue equation Eq. (4.6) becomes

$\langle 0| \mathbf{K}=\mathbf{0}$

or

$$
\begin{aligned}
& -x_{1} k_{1}+x_{2} k_{12}+\cdots+x_{n} k_{1 n}=0 \\
& x_{1} k_{21}-x_{2} k_{2}+\cdots+x_{n} k_{2 n}=0 \\
& \cdots \\
& x_{1} k_{n 1}+x_{2} k_{n 2}+\cdots-x_{n} k_{n}=0
\end{aligned}
$$

using Eq. (B.2), Eq. (B.4) becomes

$$
\begin{aligned}
& \left(x_{2}-x_{1}\right) k_{12}+\cdots+\left(x_{n}-x_{1}\right) k_{1 n}=0 \\
& \left(x_{1}-x_{2}\right) k_{21}+\cdots+\left(x_{n}-x_{2}\right) k_{2 n}=0 \\
& \cdots \\
& \left(x_{1}-x_{n}\right) k_{n 1}+\left(x_{2}-x_{n}\right) k_{n 2}+\cdots=0
\end{aligned}
$$

and therefore $x_{1}=x_{2}=\cdots=x_{n}$. The overall coefficient of $\langle 0|$ comes from the normalization condition, Eq. (4.7). The form of this eigenvector, together with Eq. (4.7), also implies that all eigenkets with non-zero eigenvalues obey $\sum_{j} x_{i j}=0=\langle 0 \mid i\rangle$.

\section{References}

[1] M.J. Pilling, P.W. Seakins, Reaction Kinetics, Oxford University Press, Oxford, 1995.

[2] J.I. Steinfeld, J.S. Francisco, W.L. Hase, Chemical Kinetics and Dynamics, second ed., Prentice-Hall, Upper Saddle River, 1999.

[3] M.S. Okino, M.L. Mavrovouniotis, Chem. Rev. 98 (1998) 391.

[4] S.J. Fraser, J. Chem. Phys. 88 (1988) 4732.

[5] A.H. Nguyen, S. Fraser, J. Chem. Phys. 91 (1989) 186.

[6] T. Turányi, A.S. Tomlin, M.J. Pilling, J. Phys. Chem. 97 (1993) 163.

[7] M.J. Davis, R.T. Skodje, J. Chem. Phys. 111 (1999) 859.

[8] J.B. Birks, Photophysics of Aromatic Molecules, WileyInterscience, London, 1970.

[9] M. Klessinger, J. Michl, Excited States and Photochemistry of Organic Molecules, VCH, New York, 1995.

[10] M.N. Berberan-Santos, J.M.G. Martinho, J. Chem. Phys. 95 (1991) 1817. 
[11] M.N. Berberan-Santos, E.J. Nunes Pereira, J.M.G. Martinho, in: D.L. Andrews, A.A. Demidov (Eds.), Resonance Energy Transfer, Wiley, Chichester, 1999.

[12] M.N. Berberan-Santos, Phys. Chem. Commun. (2000) 5.

[13] C.W. Pyun, J. Chem. Educ. 48 (1971) 194.

[14] S.P. McGlynn, T. Azumi, M. Kinoshita, Molecular Spectroscopy of the Triplet State, Prentice-Hall, Englewood Cliffs, 1969.
[15] M.N. Berberan-Santos, J.M.M. Garcia, J. Am. Chem. Soc. 118 (1996) 9391.

[16] M.N. Berberan-Santos, J.M.G. Martinho, J. Chem. Educ. 67 (1990) 375.

[17] L. Pogliani, M.N. Berberan-Santos, J.M.G. Martinho, J. Math. Chem. 20 (1996) 193. 